\title{
A low-cost chopping system and uncooled microbolometer array for ground-based astronomy
}

\author{
M. F. Rashman ${ }^{1,2}$ (D) - I. A. Steele ${ }^{1} \cdot$ S. D. Bates ${ }^{1}$. J. H. Knapen ${ }^{3,4}$
}

Received: 29 April 2020 / Accepted: 22 March 2021 / Published online: 16 April 2021

(C) The Author(s) 2021

\begin{abstract}
Mid-Infrared imaging is vital for the study of a wide variety of astronomical phenomena, including evolved stars, exoplanets, and dust enshrouded processes such as star formation in galaxies. However, infrared detectors have traditionally been expensive and it is difficult to achieve the sensitivity needed to see beyond the overwhelming mid-infrared background. Here we describe the upgrade and commissioning of a simple prototype, low-cost $10 \mu \mathrm{m}$ imaging instrument. The system was built using commercially available components including an uncooled microbolometer focal plane array and chopping system. The system was deployed for a week on the $1.52 \mathrm{~m}$ Carlos Sanchez Telescope and used to observe several very bright midinfrared sources with catalogue fluxes down to $\sim 600 \mathrm{Jy}$. We report a sensitivity improvement of $\sim 4$ mag over our previous unchopped observations, in line with our earlier predictions.
\end{abstract}

Keywords Mid-infrared · Instrumentation · Detectors · Low-cost · Microbolometer

\section{Introduction}

The mid-infrared ( $\sim 5-20 \mu \mathrm{m}$ ) background can be several orders of magnitude brighter than most astronomical sources with an $\sim 11 \mu \mathrm{m}$ surface brightness of about $-3 \mathrm{mag} / \mathrm{sq}$ arcsec $[4,22]$. This overwhelming thermal background is combined with high spatial and temporal variability of sky emission, which varies unpredictably on sub-second timescales due to air turbulence in the optical path [1], and telescopic emissions, which vary on sub-minute timescales. As a result, observing

M. F. Rashman

mfrashman@ucdavis.edu

1 Astrophysics Research Institute, Liverpool John Moores University, Liverpool, UK

2 Physics and Astronomy, University of California, Davis, California, USA

3 Instituto de Astrofísica de Canarias, Vía Láctea S/N, La Laguna, E-38205, Spain

4 Departamento de Astrofísica, Universidad de La Laguna, La Laguna, E-38206, Spain 
at these wavelengths is very challenging, particularly for ground-based facilities. Infrared-optimised telescopes must deploy a chopping/nodding regime frequently during observations to reach the fundamental photon shot-noise limit of the thermal background. These differential observations must occur at a rate faster than the background fluctuations. Chopping involves tilting either the secondary or a foreoptics mirror between on- and off-axis positions at a frequency of $\sim 10-50 \mathrm{~Hz}$ [29]. Subtraction of images taken at these two positions improves the background. Telescopic emission is not uniform and the on- and off-axis chop positions have different optical paths. This results in telescopic emission residuals in chop-subtracted images. These residuals are removed by nodding, where the telescope is slewed to an off-axis position and the chop sequence repeated, at a frequency of $\sim 0.1 \mathrm{~Hz}$ [29].

Quantitative mid-IR astronomy began in the 1960's (e.g. [21]) using heavily cooled single-element bolometric detectors on small telescopes. Since the 1970s, more complex and specialised mid-IR detectors have been successfully deployed on spaceborne and high-altitude facilities on telescopes such as the Stratospheric Observatory for Infrared Astronomy (SOFIA) (EXES; [35], FORCAST; [13]) and the Kuiper Airborne Observatory [5]. Several high-profile, space-based and high-altitude surveys have also been conducted in the mid-IR, using the Wide-field Infrared Survey Explorer (WISE) [42], Infrared Astronomical Satellite (IRAS) [27], Akari [25], the Infrared Telescope in Space (IRTS) [24], The European Large Area ISO Survey [17], Midcourse Space Experiment (MSX) [8], SPITZER (IRAC; [9]), and the Revised Air Force Geophysical Laboratory (RAFGL) [31].

High sensitivity, multi-pixel mid-IR detectors have been in operation at large ground-based telescopes since the 1990's, such as the $3 \mathrm{~m}$ IRTF (MIRSI; [7]), the 6.5 m MMT (MIRAC; [14]), the $8 \mathrm{~m}$ Gemini (Michelle; [11], T-ReCS; [38], TEXES; [18]) and VLT (VISIR; [19], MIDI; [20]), the $8.2 \mathrm{~m}$ Subaru (Spectro-Cam; [12], COMICS; [16]) and the 10.6 m Gran Telescopio Canarias (GTC) (CanariCam; [28]). These large facility, mid-infrared systems use $\mathrm{Si}$ :As impurity band conduction arrays for their detectors. These instruments are not readily adapted for use on smaller $1-2 \mathrm{~m}$ class telescopes due to the high costs associated with their extensive cooling systems and specialist detector systems. It is generally considered impossible to reach the sensitivity needed to observe all but the brightest mid-IR sources, with an uncooled, simply designed, low-cost instrument.

Uncooled microbolometers are designed to detect incident radiation at wavelengths in the range $7.5-14 \mu \mathrm{m}$ as changes in resistance across their focal plane array (FPA). Typically employing vanadium oxide (VOx) or amorphous silicon (a-Si), microbolometer technology was originally developed for defence, security and industrial applications in the 1980s. These systems are now integrated into commercial 'off the shelf' (COTS) thermal imaging cameras for a price of less than $10000 \mathrm{GBP}$, and can deliver noise-equivalent temperature differential (NEdT) measurements of $<200 \mathrm{mK}$ [3], although our previous lab-based experiments have recorded NEdT measurements of $\sim 60 \mathrm{mK}$ [33]. Apart from some applications on small $(<200 \mathrm{~mm})$ telescopes [36, 40] and high-altitude experiments [39], these systems had never been adapted for use in astronomy.

Mid-IR radiation is an effective tracer of star and planet forming regions, evolved stars, Solar System objects and galaxies. Successful use of widely-available, 
microbolometer technology on $1-2 \mathrm{~m}$ class telescopes would expand the availability of mid-IR observing by reducing the costs associated with the development and maintenance of ground-based infrared instruments. A system of this kind would never be competitive with the cooled systems employed at the larger facilities listed above, however it could prove beneficial for monitoring bright variable systems and Solar System planetesimals like asteroids. One current high-profile example of this is the recent optical dimming event of red supergiant Alpha Orionis, in which $\mathrm{N}$ band observations were employed to aid in the interpretation of such an event [10]. Alongside applications in time domain astrophysics, a greater availability of midIR observing facilities on smaller telescopes could provide an invaluable resource to train students and early career researchers on mid-IR observing techniques and data analysis.

Recognising the potential for astronomical applications of this technology, we developed and commissioned a low-cost, COTS system for use on the $2 \mathrm{~m}$ Liverpool Telescope (LT) using germanium re-imaging foreoptics to collimate the light onto the detector. The prototype was described in [34] and is a small $(<1 \mathrm{~kg})$, passively cooled, $N$-band $(10 \mu \mathrm{m})$ imager adapted from a FLIR Tau camera with a $640 \times 512$ VOx microbolometer array. The instrument was successfully deployed on the LT [37] in January 2019 and we obtained a series of observations of very bright mid-infrared objects and the total lunar eclipse. From these observations, we recorded a sensitivity limit of $\sim 7 \times 10^{3} \mathrm{Jy}$ for a single, $\sim 0.11$ second exposure. This corresponds to a $3 \sigma$ detection limit for only the brightest mid-infrared objects, such as IRC+10216 [26] and Mars. A theoretical sensitivity for a stack of exposures totalling an integration time of $\sim 60$ seconds was calculated to have a detection limit for objects 163 times fainter than IRC+10216 $\left(\sim 3 \times 10^{2} \mathrm{Jy}\right)$. However, we were unable, given the overwhelming and rapidly variable background, to reach that limit.

In this paper, we describe an upgrade to our prototype instrument to include a chopping mirror in the foreoptics. We present the result of a week-long programme of observations on the Carlos Sanchez Telescope (TCS) [15] at the Observatorio del Teide, designed to test the chopping mirror and implement an effective nodding regime.

\section{Optical design}

The basic instrument is described in detail in [34]. Our goal here is to improve instrument sensitivity with the integration of a chopping mirror to the foreoptics, which are comprised of two anti-reflection (AR) coated germanium lenses, a $50 \mathrm{~mm}$ diameter field lens and $25 \mathrm{~mm}$ diameter collimator lens with $76 \mathrm{~mm}$ separation. In addition to this, we carried out our observations on the TCS which is optimised for infrared imaging. The TCS is an f/13.8 telescope with a Dall-Kirkham design. The TCS is comprised of a thin $1.52 \mathrm{~m}$ concave ellipsoid primary mirror and an undersized $0.37 \mathrm{~m}$ convex spherical secondary mirror, with minimal baffling. These features act to lower the thermal background compared to a conventional optical telescope such as the LT. The TCS has a $10 \mu \mathrm{m}$ diffraction limit of 1.66 arcsec. To rescale the image to include a chopping mirror in the foreoptics and determine the appropriate plate scale 
for use on the TCS, a ray tracing analysis for on- and off-axis light was conducted. The updated design can be seen in Fig. 1.

This updated optical design does not change the position of the field and collimator lenses with respect to each other and the detector. However, the new optical design accounts for the change in telescope and the inclusion of the chopping mirror at $45^{\circ}$. Computed spot diagrams for on-axis and $\pm 2.7^{\prime}$ off-axis rays show no deviation from the spot diagrams produced for the base instrument as described in [34], with offaxis aberration still present. However, the $80 \%$ geometric encircled energy (GEE) of system is changed and a diameter of $<1.7$ arcsec for on-axis rays and $<3.6$ arcsec for $\pm 2.7^{\prime}$ off-axis rays is computed. Assuming a 2D Gaussian profile, this corresponds to a spatial full width half maximum (FWHM) of $<1.1$ and $<2.3$ arcsec for on- and off-axis rays respectively. From this, it was predicted that 2 pixels would sample the diffraction limit, with a plate scale of $0.96^{\prime \prime}$, for on-axis rays, with slightly worse performance off-axis. The imaging field of view (FOV) is reduced to a circular aperture of 500 pixel diameter due to the likely presence of an aperture stop within the camera lens (see [34] for further discussion).

\section{Predicted sensitivity}

In order to predict the potential sensitivity of the system, we make the assumption that the nodding and chopping system will remove all variability in the sky and telescope emission, and that the sensitivity is therefore dominated by the ability of the detector to discriminate a temperature rise (its NEDT) against a high static background flux.

Assuming an $N$-band sky magnitude of $-3.0 /$ sq arcsec $[4,6,22]$ and pixel scale of 0.5 arcsec per pixel we calculate an incident power per pixel on the detector of

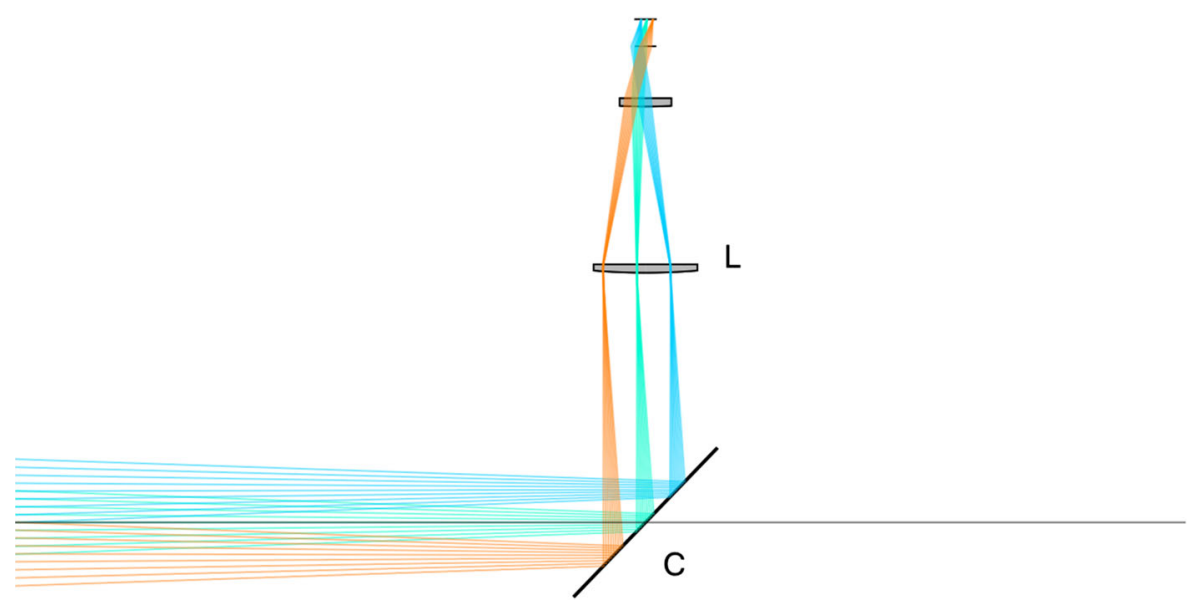

Fig. 1 Side-view ray trace of the instrument, as mounted on the TCS, for on- and off-axis beams of 2.7 arcmin. This zoomed-in portion shows how the chopping mirror $\mathbf{C}$, acts to direct the rays perpendicular to the optical path and onto the $50 \mathrm{~mm}$ diameter field lens $\mathbf{L}$, that sits at the front of the existing foreoptics (see [34]) 
$5.8 \times 10^{-11} \mathrm{~W}$ integrated over the detector wavelength range. Similarly assuming a telescope temperature of $293 \mathrm{~K}$ and an emissivity of 0.1 , we predict a wavelengthintegrated incident power per pixel on the detector of $1.2 \times 10^{-8} \mathrm{~W}$.

The effect of the total of the sky and telescope incident power on the detector pixel temperatures can then be modelled as:

$$
P=k T^{4},
$$

where $T$ is the recorded temperature of a pixel and $k$ is a calibration constant for the detector which can be calculated from the recorded background temperature of sky images $(\sim 253 K)$ [32].

If $\delta P$ is the additional power that would fall on detector from a star which will raise the detector temperature by the NEDT then we can modify (1) as:

$$
P+\delta P=k(T+N E D T)^{4} .
$$

For our measured NEDT of $60 \mathrm{mK}$, this predicts an excess power requirement to detect an object of $\delta P \sim 1.1 \times 10^{-11} \mathrm{~W}$. Assuming this power is spread over 9 pixels (i.e. within a circle of FWHM 1.5 arcsec given the plate scale), this implies a sensitivity limit of $\sim 450 J y$ (corresponding to a magnitude $N \sim-2.8$ ).

\section{Mechanical design}

The instrument was constructed as modelled in Fig. 2. The field and collimator lenses were housed in an anodised aluminium tube of length $114 \mathrm{~mm}$ and diameter $50 \mathrm{~mm}$. The camera mounte§d to this tube via a custom adaptor milled from low-grade aluminium. Rather than integrate these parts into the main body of the instrument, they were left self-contained and the instrument body was designed to have two options for mounting; (1) at the straight-through position, used only when the chopping mirror is removed from the light path, to aid in initial setup of the instrument, (2) perpendicular to the direction of rays entering the instrument. The Edmund Optics $75 \times 100 \mathrm{~mm}$ gold coated, $4-6 \lambda$ chopping mirror (reflectance at $45^{\circ}: R_{\text {avg }}>96 \%$ for 3-8 $\mu \mathrm{m}$ and $10-14 \mu \mathrm{m}$, dropping to $R_{\text {avg }}>75 \%$ for $8-10 \mu \mathrm{m}$ ) is located at position (3) and sits atop a Thorlabs ELL18K 50.0mm piezoelectric rotation stage which operates with a home angle of $45^{\circ}$ and directs the light in the perpendicular direction onto the field lens. A $1^{\circ}$ angular tilt of the chopping mirror results in a relative chop size of $\sim 44$ pixels on the detector and $22 \operatorname{arcsec}$ on sky. The rotation stage is recommended to be mounted in a horizontal orientation, especially when carrying a load such as the chopping mirror. However, the varying elevation of telescopes means that we could not guarantee the platform would deployed in horizontally during observations and it is worth noting that this can cause a reduction in performance. A Thorlabs DCC3240M optical CMOS camera (4) was included to provide the possibility of dual wavelength data acquisition or to act as a guide camera. This can be deployed with a $25.0 \times 23.5 \times 1.0 \mathrm{~mm}$ polished germanium optical window acting as a dichroic on a Thorlabs $60 \mathrm{~mm}$ Piezo Elliptical Linear Stage (5). Low-cost, rapid-prototyping techniques including $3 \mathrm{D}$ printing were used to create mounts for the optomechanical components. All parts were installed on a $1 \mathrm{~cm}$ thick aluminium 


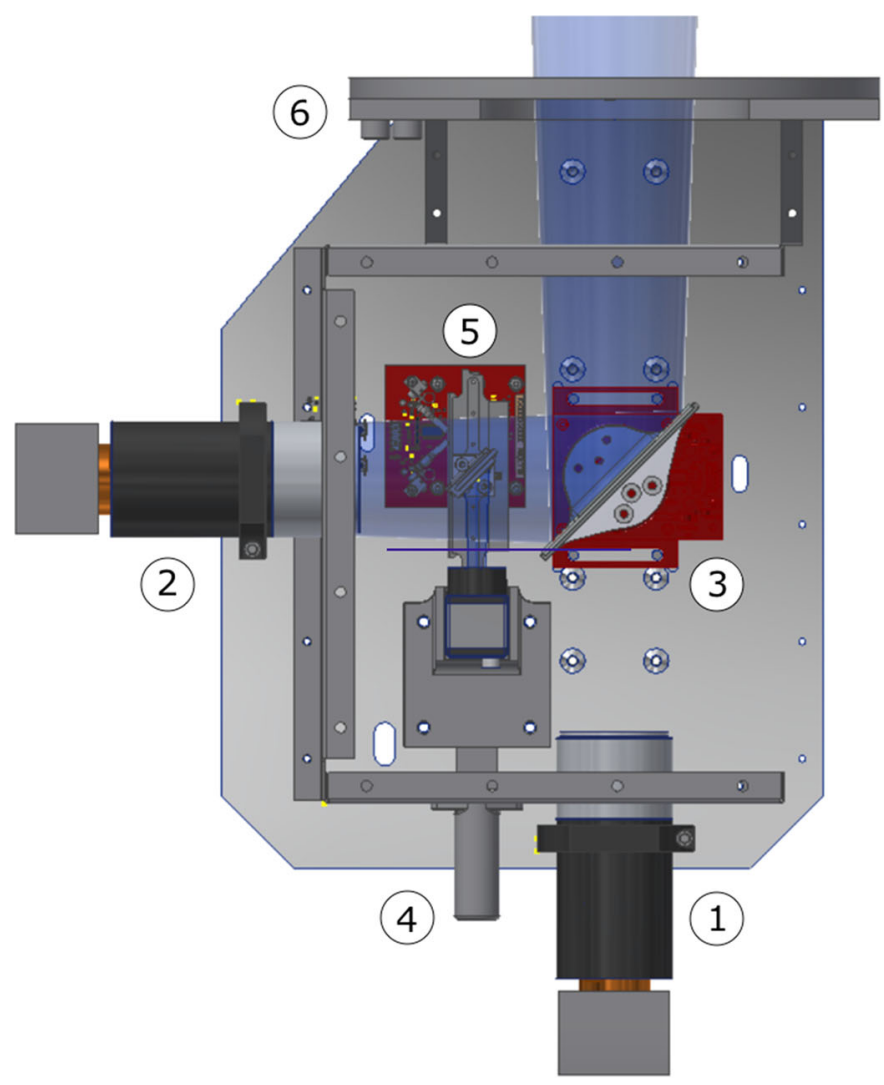

$0.4 \mathrm{~m}$

Fig. 2 The mechanical design of the instrument. Main components are numbered as follows; (1) first mount position for camera and foreoptics, (2) second mount position for camera and foreoptics, (3) chopping mirror on rotational platform, (4) Optical camera on adjustable mount for focusing, (5) deployable silver mirror on sliding platform, (6) mounting plate

baseplate with a folded aluminium cover. The instrument was installed at $\sim 0.84 \mathrm{~m}$ from the TCS mounting flange using the mounting plate (6). When constructed, the prototype has dimensions of $\sim 35 \times 40 \times 20 \mathrm{~cm}$ and a weight of $6.0 \pm 0.05 \mathrm{~kg}$. The instrument was constructed without dust/dirt protection due to the brevity of the testing period. However, introducing rubber seals around the mounting points and/or a slight positive air pressure inside the instrument would be beneficial in protecting the mechanical components and optics from dust/dirt for a longer period of deployment on a telescope.

The instrument was paired with a Beelink J45 Mini PC running Xubuntu. This CPU allowed for all parts to be controlled remotely. Software was written to trigger data acquisition with each exposing period lasting for $\sim 2$ minutes, with images downloading at a frequency of $\sim 9 \mathrm{~Hz}$. The python Pyserial module was employed to communicate with mechanical platforms. This was integrated into the exposing routine for smooth operation of the instrument and to aid in identifying the mirror 
position for each exposure. A complementary python pipeline was designed to reduce the raw data. This pipeline sorts input observations into chopped and unchopped subsets. Any observations taken while the chopping mirror is in motion are identified and removed. Chopped exposures are then subtracted from subsequent unchopped exposures. The resulting chop-subtracted observations are then mean-stacked to output one master observation for each exposing period.

\section{Commissioning and on-sky testing}

The instrument was installed on the TCS on 2019 Sep. 10. It was deployed over eight nights, although three nights were lost to rain. The performance of the instrument for dual-wavelength acquisition was not robustly tested due to the time lost to weather. However, the optical system proved useful for pointing.

\subsection{Background reduction}

All observations were taken with a chop throw of $\sim 11^{\prime \prime}$, at a frequency of $1 \mathrm{~Hz}$. The chop frequency was limited by the speed and positional accuracy of the rotational platform. Although Thorlabs state the piezoelectric rotation stage has an operational velocity of 430 degrees per second with a homing and bidirectional repeatability of 0.1 and $0.05^{\circ}$ respectively, and a bidirectional accuracy of $0.4^{\circ}$, we found that when moving the platform through small angles of $0.5-1^{\circ}$ at frequencies greater than $1 \mathrm{~Hz}$,we were unable to achieve sufficient positional repeatability for chopping the mirror. The platform deploys coarse $(0.5$ to $1.0 \mathrm{~mm})$ and fine $(1.0 \mu \mathrm{m})$ tuning for positioning and we observed that these mechanisms occured on slower timescales, with each position needing to be separated by at minimum $\sim 1$ s to settle between movement. When chopping at a higher frequency, the platform could not settle between each movement and these small changes in position would translate into a large degree drift for the duration of the observation. This limitation imposed on the chopping frequency by the positional tuning was observed in both lab (horizontal on an optical bench) and observing (with varying elevation and orientation) conditions however it may have been exacerbated by non-optimal use of the platform. We observed no sign of electromagnetic interference from the piezo driver for the rotation platform however this was not robustly tested. We expect any unwanted signal from the rotational platform would be overwhelmed by the general thermal infrared background in the optical system and the uncooled detector housing.

The instrument was initially intended for use with a standard ABBA nod pattern, where chop sequences occur at nod positions $\mathrm{A}$ and $\mathrm{B}$, and the final observation is produced from the subtraction pattern (A-B)-(B-A). This would produce an observation with four images of the object; two negative and two positive. However, the positional accuracy of the offsets of the TCS used to implement nodding was poor. Figure 3 a shows a reduced observation taken with an ABBA nod pattern. Six object images are present as a result of the lack of alignment between positive and negative sources in subtracted chop images. There is also an overwhelming residual background as a result of unsuccessful subtraction of telescope emissions. An ABCD 


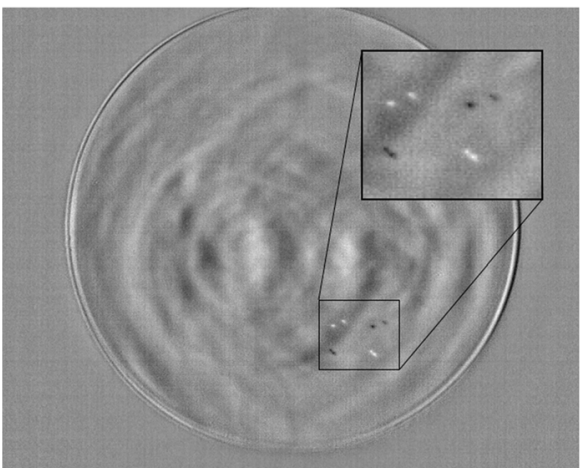

a

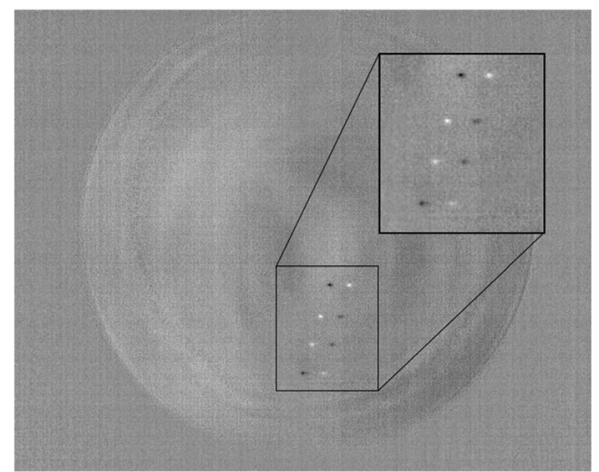

b

Fig. 3 The positional accuracy of the telescope affects the performance of nod patterns. a Standard ABBA nod pattern that relies on accurate re-positioning of the telescope to produce two positive and two negative images of the object. b ABCD nod pattern where the telescope is nodded through 4 different positions in $15^{\prime \prime}$ increments. This results in an increased number of positive and negative images of the source but removes the need for accurate positioning

pattern was thus used to remove the need for accurately re-positioning the telescope. In an ABCD nod pattern, the telescope is nodded perpendicular to the chop direction through four different positions in $15^{\prime \prime}$ increments. Chop sequences occur at all positions and the final observation is produced from an (A-B)-(C-D) subtraction pattern. An example of an observation using the ABCD nod pattern can be seen in Fig. 3b. Overall, nodding produced variable results when it came to removing telescopic emissions. The nod period of $\sim 2$ minutes was determined by the offset speed of the telescope and was much longer than nod periods deployed at other facilities. Due to weather constraints, a shorter nod period was not tested. However, it is likely that the background reduction can be improved by reducing the nod period to $<1$ minute.

An example of full background reduction can be seen in Fig. 4. Figure 4a shows a typical single raw observation taken in one exposing period with the instrument. The FOV is limited to the circular aperture in the centre. Emission from the telescope is visible as the spider-like structure. Figure $4 \mathrm{~b}$ is the output from the chop reduction pipeline which we used to create and stack $\sim 300$ chop-subtracted observations. Figure $4 \mathrm{c}$ and $\mathrm{d}$ are the result of subtracting nod positions for this exposure. Observations of the brightest source NML Cyg were fitted with Gaussian profiles that have a FWHM in the range of $1.0-2.0$ arcsec. The lower values are consistent with the spatial FWHM obtained from the optical design and the diffraction limit. Many of the combined source images with higher FWHM appear to be elliptical in shape. This could be a result of the optics and the off-axis aberration that is inherent in the design. However, there are several other processes involved in obtaining the chop/nod reduced observations that could have contributed to the ellipticity of sources. The telescope tracking was poor and over the course of a chop cycle, may have drifted 


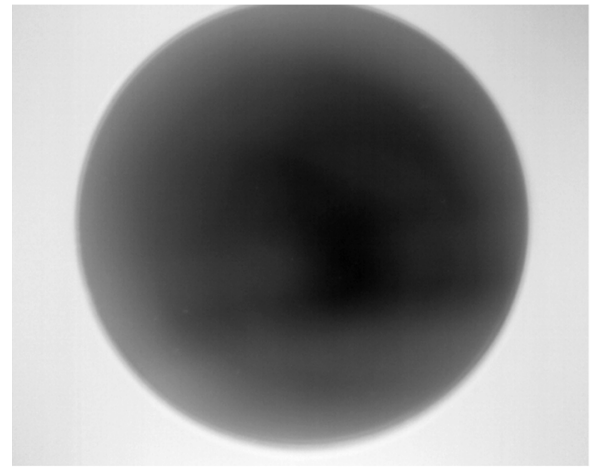

a

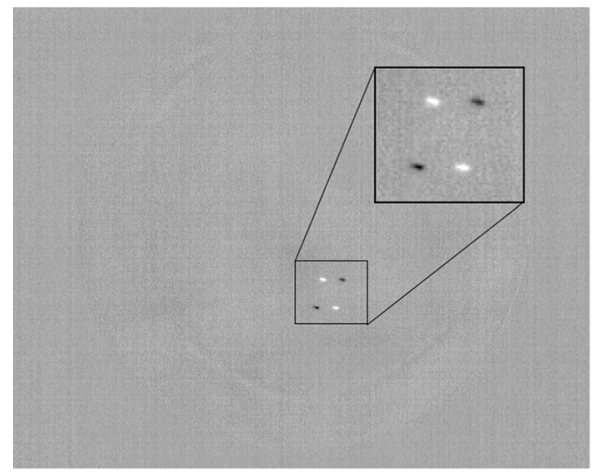

C

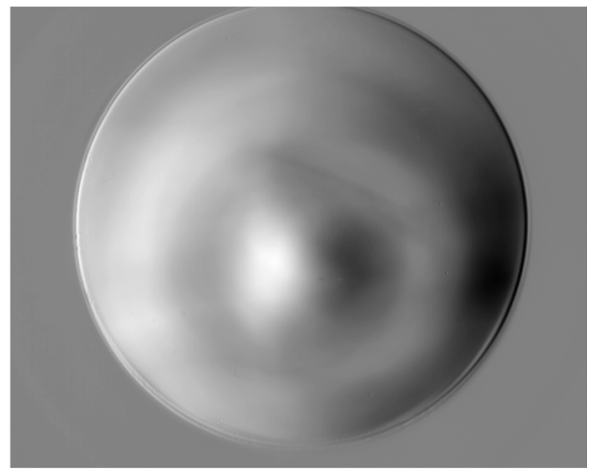

b

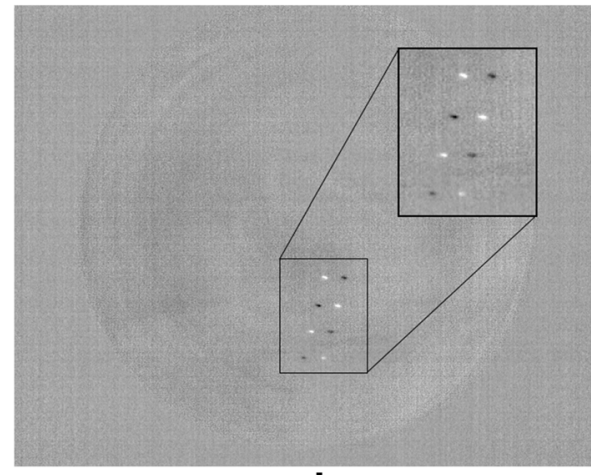

d

Fig. 4 An example of background reduction after upgrading the instrument to include a chopping mirror and taking observations with an ABCD nod regime. a Single raw observation of the red supergiant NML Cygni, taken with the TCS on 2019 September 14. b 300 stacked, chop-subtracted, observations of NML Cygni. c Subtraction of stacks from nod positions A and B. d Full ABCD chop/nod reduction. The imaging field of view is limited to the circular area in the centre

sufficiently that the sources in early and late, chopped and unchopped, observations were no longer aligned. Likewise, the positional accuracy of the rotational platform on which the chopping mirror was mounted could have been insufficient.

\subsection{Sensitivity}

To determine the sensitivity of the system, observations were taken of bright $11 \mu \mathrm{m}$ sources selected from the RAFGL catalogue [31]. In [34] observations of IRC+10216 were determined to be at the sensitivity limit of the instrument as no fainter sources were detected. IRC+10216 is a known variable and listed with an RAFGL $N$-band magnitude of -7.7. However, it was unobservable from the Observatorio del Teide in September 2019, therefore no direct comparisons could be made for the new instrument configuration. Instead, 13 sources were selected from the RAFGL catalogue 
for observation, of which 10 could be detected by eye in the chop/nod reduced data. Table 1 lists these sources by their common identifier. Any objects marked by an asterisk were not confirmed in the data. Each object was cross-referenced across three other mid-infrared catalogues; the WISE all-sky source catalogue [42], the IRAS point source catalogue v2.1 [2] and the MSX catalogue [8]. The objects are also listed against their type as listed in the SIMBAD astronomical database [41], with all sources likely to have at least some variability.

The measured counts of all ten observed sources were compared to their respective catalogue fluxes, with RAFGL magnitudes converted to flux using the conversion factor given by [30]. Measured counts were determined from averaging the total signal measured in an aperture for all positive sources present in chop/nod-subtracted observations. Aperture size was selected using a curve of growth technique to maximise signal to noise ratio. Several of the sources show significant variability between catalogue values so a least squares regression was conducted for sources whose catalogue fluxes are within $30 \%$ of each other. Although a naive estimate of linearity, based on a select few sources, the positive linear trend obtained from the least squares regression indicates the instrument is likely functioning as intended. However, a further factor of 10x in sensitivity would be needed to observe typical bright, non-variable standard stars, such as Vega, to allow a proper calibration.

Table 1 The 13 bright $11 \mu \mathrm{m}$ sources selected for observation from the RAFGL catalogue

\begin{tabular}{|c|c|c|c|c|c|}
\hline \multirow[t]{2}{*}{ Identifier } & \multicolumn{5}{|c|}{$11 \mu \mathrm{m}$ Flux Jy $11.6 \mu \mathrm{m}$ Flux Jy $12 \mu \mathrm{m}$ Flux Jy $12.13 \mu \mathrm{m}$ Flux Jy Type } \\
\hline & (RAFGL) & (WISE) & (IRAS) & (MSX) & \\
\hline NML Cyg & $6.38 \mathrm{e}+03$ & $4.65 e+02$ & $2.812 \mathrm{e}+03$ & & Red supergiant \\
\hline R Cas & $1.60 \mathrm{e}+03$ & $3.89 \mathrm{e}+02$ & $1.34 \mathrm{e}+03$ & & Variable Star (Mira Cet) \\
\hline IRC +10420 & $1.60 \mathrm{e}+03$ & $3.68 \mathrm{e}+02$ & $1.35 \mathrm{e}+03$ & $1.2925 \mathrm{e}+03$ & Post-AGB Star (proto-PN) \\
\hline$\alpha$ Herculis & $1.33 \mathrm{e}+03$ & $4.24 \mathrm{e}+02$ & & & Double or multiple \\
\hline$\chi \mathrm{Cyg}$ & $1.22 \mathrm{e}+03$ & $4.20 \mathrm{e}+02$ & $1.69 \mathrm{e}+03$ & $1.7482 \mathrm{e}+03$ & S Star \\
\hline IRC - 10529* & $* 1.01 \mathrm{e}+03$ & $3.07 \mathrm{e}+02$ & $1.26 \mathrm{e}+03$ & & $\mathrm{OH} / \mathrm{IR}$ star \\
\hline W Aql & $1.01 \mathrm{e}+03$ & $3.41 \mathrm{e}+02$ & $1.58 \mathrm{e}+03$ & & S Star \\
\hline IRC +10365 & $8.41 \mathrm{e}+02$ & $3.06 \mathrm{e}+02$ & $7.20 \mathrm{e}+02$ & & Variable Star (Mira Cet) \\
\hline V Cyg & $8.41 \mathrm{e}+02$ & $3.38 \mathrm{e}+02$ & $6.65 e+02$ & $8.9260 \mathrm{e}+02$ & Carbon Star \\
\hline $\mathrm{IRC}+20370$ & $8.41 \mathrm{e}+02$ & $3.09 \mathrm{e}+02$ & $5.34 \mathrm{e}+02$ & & Carbon Star \\
\hline e Aql* & $6.38 \mathrm{e}+02$ & $2.71 \mathrm{e}+01$ & $2.79 \mathrm{e}+01$ & & Variable \\
\hline GY Aql & $5.82 \mathrm{e}+02$ & $3.04 \mathrm{e}+02$ & $4.61 \mathrm{e}+02$ & & Variable Star (Mira Cet) \\
\hline X Oph* & $5.82 \mathrm{e}+02$ & $2.82 \mathrm{e}+02$ & $4.09 \mathrm{e}+02$ & & Variable Star (Mira Cet) \\
\hline
\end{tabular}

All objects are listed by their common identifier with those marked by an asterisk (*) confirmed as not detected in the data. All objects have been cross referenced across four catalogues (RAFGL, WISE, IRAS and MSX). RAFGL and WISE flux values have been converted from their respective catalogue magnitudes. Sources are either identified as known variables or likely to display some variability 


\section{Discussion and conclusion}

In this paper we have presented further development of our prototype instrument that adapts mid-IR uncooled microbolometer technology for use on ground-based telescopes in the 1-2 m class. To improve on sensitivity, a chopping module was designed and implemented into the foreoptics of the system to be deployed alongside a telescope nodding routine. This addition was built from commercially available units, at a low cost, much like the rest of the instrument. The instrument was tested on the TCS over 8 days in 2019 September. A plate scale of $0.5^{\prime \prime}$ per pixel on the TCS was recorded, indicating that the image is slightly oversampled. Varying nod patterns were tested, with an ABCD pattern selected as the best option for the positional accuracy of the telescope and system. After commissioning, a programme of observations of 13 stellar objects selected from the RAFGL catalogue was conducted.

Whilst ten sources were observed with the system, only four fulfilled a selection criteria of having catalogue values that vary by no more than $30 \%$. The faintest of these sources, according to the values recorded in RAFGL, IRAS and MSX, are V Cyg and IRC +10365 , with fluxes in the range of $\sim 600-900 \mathrm{Jy}$. This is a vast improvement on our previous observations with an unchopped system, as sources $\sim 70$ times fainter are now observable. The addition of a chop/nod regime puts the system much closer to our theoretical estimate of a sensitivity limit for the system of $\sim 450 \mathrm{Jy}$ as derived in section 3, and our previous empirical estimate of $\sim 300 \mathrm{Jy}$ based on scaling our previous Liverpool Telescope data, and is starting to approach that of bright asteroids ( 100 Jy) [23].

The offset speed of the telescope, and the positioning speed of the rotational platform limited the period at which chopping and nodding could occur. Under these circumstances, the quality of chop/nod background reduction was highly variable, with overwhelming residuals left in several reduced images. Reducing the nodding and chopping periods in particular, to frequencies more in line with those at other infrared facilities, is likely to make some improvements to the sensitivity and quality of data reduction going forward. This would require improving the positional speed and accuracy of the chopping mechanism, and by deploying the system on an infrared telescope that is optimised for fast nodding. The rotation platform used for chopping has a manufacturer estimated minimum lifetime of 600,000 rotations, which for a chopping frequency of $1 \mathrm{~Hz}$ corresponds to a minimum lifetime of $\sim 166$ hours of operation. This, alongside the observed limitations in positional repeatability and chopping frequency, makes this particular rotation platform not a long term solution for chopping. We would advise greater investment into a rotational stage. These improvements to the chopping and nodding would likely gain sensitivity improvements much closer to our previously estimated limits and a greater longevity of the instrument. It may be that further sensitivity improvements could be achieved by modestly cooling (e.g. $\delta T=-40 \mathrm{~K}$ ) the system with the addition of a low-cost thermoelectric cooler to lower system NEDT and internal thermal emission in the camera.

Acknowledgements This article is based on observations made at Telescopio Carlos Sánchez operated on the island of Tenerife by the Instituto de Astrofísica de Canarias (IAC) in the Spanish Observatorio 
del Teide. We would like to express our thanks to the telescope operator and support astronomer who aided us with these observations. This research has made use of the SIMBAD database, operated at CDS, Strasbourg, France. MFR is funded by a Liverpool John Moores University FET/SCS PhD scholarship. This research has made use of the NASA/ IPAC Infrared Science Archive, which is operated by the Jet Propulsion Laboratory, California Institute of Technology, under contract with the National Aeronautics and Space Administration. J.H.K. acknowledges financial support from the European Union's Horizon 2020 research and innovation programme under Marie Skłodowska-Curie grant agreement No 721463 to the SUNDIAL ITN network, from the State Research Agency (AEI) of the Spanish Ministry of Science and the European Regional Development Fund (FEDER) under the grant with reference PID2019-105602GBI00, from IAC project P/300724, financed by the Ministry of Science through the State Budget and by the Canary Islands Department of Economy, Knowledge and Employment, through the Regional Budget of the Autonomous Community. This project has received funding from the European Union's Horizon 2020 research and innovation programme under grant agreement No 730890. This material reflects only the authors views and the Commission is not liable for any use that may be made of the information contained therein.

Open Access This article is licensed under a Creative Commons Attribution 4.0 International License, which permits use, sharing, adaptation, distribution and reproduction in any medium or format, as long as you give appropriate credit to the original author(s) and the source, provide a link to the Creative Commons licence, and indicate if changes were made. The images or other third party material in this article are included in the article's Creative Commons licence, unless indicated otherwise in a credit line to the material. If material is not included in the article's Creative Commons licence and your intended use is not permitted by statutory regulation or exceeds the permitted use, you will need to obtain permission directly from the copyright holder. To view a copy of this licence, visit http://creativecommons.org/licenses/by/4.0/.

\section{References}

1. Absil, O., Bakker, E., Schöller, M., Gondoin, P.: Thermal background fluctuations at 10 micron measured with vlti/midi. Proceedings of SPIE - The International Society for Optical Engineering. https://doi.org/10.1117/12.549311 (2004)

2. Beichman, C.A., Neugebauer, G., Habing, H.J., Clegg, P.E., Chester, T.J.: Infrared astronomical satellite (iras) catalogs and atlases. volume 1: explanatory supplement 1 (1988)

3. Benirschke, D., Howard, S.: Characterization of a low-cost, commercially available, vanadium oxide microbolometer array for spectroscopic imaging. Optical Engineering 56, 4, 1-4, 4 (2017)

4. Bertero, M., Boccacci, P., Robberto, M.: Wide-field imaging at mid-infrared wavelengths: reconstruction of chopped and nodded data. Publ. Astron. Soc. Pac. 112(774), 1121-1137 (2000)

5. Cameron, R.M.: NASA'S 91-cm airborne telescope. Sky and Telescope 52, 327 (1976)

6. Cohen, M., Walker, R.G., Carter, B., Hammersley, P., Kidger, M., Noguchi, K.: Spectral irradiance calibration in the infrared. $\mathrm{x}$. a self-consistent radiometric all-sky network of absolutely calibrated stellar spectra. The Astronomical Journal 117(4), 1864-1889 (1999)

7. Deutsch, L.K., Hora, J.L., Adams, J.D., Kassis, M.: MIRSI: a mid-infrared spectrometer and imager. In: Iye, M., Moorwood, A.F.M. (eds.) Proc. SPIE, Society of Photo-Optical Instrumentation Engineers (SPIE) Conference Series, vol. 4841, pp. 106-116 (2003). https://doi.org/10.1117/12.461436

8. Egan, M.P., Price, S.D.: The MSX infrared astometric catalog. Astronomical Journal 112, 2862 (1996)

9. Fazio, G.G., Hora, J.L., Willner, S.P., Stauffer, J.R., Ashby, M.L., Wang, Z., Tollestrup, E.V., Pipher, J.L., Forrest, W.J., McCreight, C.R., Moseley, S.H., Hoffmann, W.F., Eisenhardt, P., Wright, E.L.: Infrared array camera (IRAC) for the space infrared telescope facility (SIRTF). In: Fowler, A.M. (ed.) Proc. SPIE, Society of Photo-Optical Instrumentation Engineers (SPIE) Conference Series, vol. 3354, pp. 1024-1031 (1998)

10. Gehrz, R.D., Marchetti, J., McMillan, S., Procter, T., Zarling, A., Bartlett, J., Smith, N.: Betelgeuse remains steadfast in the infrared. The Astronomer's Telegram 13518, 1 (2020)

11. Glasse, A.C., Atad-Ettedgui, E.I., Harris, J.W.: Michelle midinfrared spectrometer and imager. In: Ardeberg, A.L. (ed.) Proc. SPIE, Optical Telescopes of Today and Tomorrow, vol. 2871, pp. 11971203 (1997)

12. Hayward, T.L., Miles, J.E., Houck, J.R., Gull, G.E., Schoenwald, J.: Spectrocam-10: a 10-um spectrograph/camera for the hale telescope. In: Fowler, A.M. (ed.) Proc. SPIE, Society of Photo-Optical 
Instrumentation Engineers (SPIE) Conference Series, vol. 1946, pp. 334-340 (1993). https://doi.org/ $10.1117 / 12.158686$

13. Herter, T.L., Adams, J.D., Gull, G.E., Schoenwald, J., Keller, L.D., Pirger, B.E., Henderson, C.P., Stacey, G.J., Nikola, T., De Buizer, J.M., Vacca, W.D., Ennico, K.: FORCAST: a midinfrared camera for SOFIA. Journal of Astronomical Instrumentation 7(4), 1840005-451 (2018). https://doi.org/10.1142/S2251171718400056

14. Hoffmann, W.F., Fazio, G.G., Ki, S., Hora, J.L., Deutsch, L.K.: MIRAC: a mid-infrared array camera for astronomy. In: Fowler, A.M. (ed.) Proc. SPIE, Society of Photo-Optical Instrumentation Engineers (SPIE) Conference Series, vol. 1946, pp. 449-460 (1993). https://doi.org/10.1117/12.158697

15. Jones, T.: El Teide and the flux collector. Infrared astronomy on Tenerife. Journal of the British Astronomical Association 88, 257-266 (1978)

16. Kataza, H., Okamoto, Y., Takubo, S., Onaka, T., Sako, S., Nakamura, K., Miyata, T., Yamashita, T.: COMICS: the cooled mid-infrared camera and spectrometer for the Subaru telescope. In: Iye, M., Moorwood, A.F. (eds.) Proc. SPIE, Society of Photo-Optical Instrumentation Engineers (SPIE) Conference Series, vol. 4008, pp. 1144-1152 (2000). https://doi.org/10.1117/12.395433

17. Kessler, M.F., Steinz, J.A., Anderegg, M.E., Clavel, J., Drechsel, G., Estaria, P., Faelker, J., Riedinger, J.R., Robson, A., Taylor, B.G., Ximenez de Ferran, S.: The Infrared Space Observatory (ISO) mission. A\&A 500, 493-497 (1996)

18. Lacy, J.H., Richter, M.J., Greathouse, T.K., Jaffe, D.T., Zhu, Q., Knez, C.: TEXES: sensitive and versatile spectrograph for mid-infrared astronomy. In: Iye, M., Moorwood, A.F.M. (eds.) Proc. SPIE, Society of Photo-Optical Instrumentation Engineers (SPIE) Conference Series, vol. 4841, pp. 15721580 (2003). https://doi.org/10.1117/12.461194

19. Lagage, P.O., Durand, G.A., Lyraud, C., Rio, Y., Pel, J.W., de Haas, J.C.: Final design of VISIR: the mid-infrared imager and spectrometer for the VLT. In: Iye, M., Moorwood, A.F. (eds.) Proc. SPIE, Optical and IR Telescope Instrumentation and Detectors, vol. 4008, pp. 1120-1131 (2000)

20. Leinert, C., Graser, U., Przygodda, F., Waters, L., Perrin, G., Jaffe, W., Lopez, B., Bakker, E., Böhm, A., Chesneau, O., Cotton, W., Damstra, S., de Jong, J., Glazenborg-Kluttig, A., Grimm, B., Hanenburg, H., Laun, W., Lenzen, R., Ligori, S., Mathar, R., Meisner, J., Morel, S., Morr, W., Neumann, U., Pel, J.W., Schuller, P., Rohloff, R.R., Stecklum, B., Storz, C., von der Lühe, O., Wagner, K.: Midi the $10 \mu \mathrm{m}$ instrument on the vlti. Astrophysics \& Space Science 286(1), 73-83 (2003)

21. Low, F.J.: Low-temperature germanium bolometer. J. Opt. Soc. Am. 51(11), 1300-1304 (1961)

22. Mason, R., Wong, A., Geballe, T., Volk, K., Hayward, T., Dillman, M., Fisher, R.S., Radomski, J.: Observing conditions and mid-IR data quality. In: Proc. SPIE, Society of Photo-Optical Instrumentation Engineers (SPIE) Conference Series, vol. 7016, p. 70161 Y (2008)

23. Müller, T.G., Lagerros, J.S.V.: Asteroids as calibration standards in the thermal infrared for space observatories. Astron. Astrophys. 381, 324-339 (2002)

24. Murakami, H., Bock, J., Freund, M.M., Guo, H., Hirao, T., Lange, A.E., Matsuhara, H., Matsumoto, T., Matsuura, S., McMahon, T.J., Murakami, M., Nakagawa, T., Noda, M., Noguchi, K., Okuda, H., Okumura, K., Onaka, T., Roellig, T.L., Sato, S., Shibai, H., Tanabe, T., Watabe, T., Yagi, T., Yajima, N., Yui, M.: The infrared telescope in space (IRTS). Astrophys. J. 428, 354 (1994). https://doi.org/10.1086/174246

25. Murakami, H., Baba, H., Barthel, P., Clements, D.L., Cohen, M., Doi, Y., Enya, K., Figueredo, E., Fujishiro, N., Fujiwara, H., Fujiwara, M., Garcia-Lario, P., Goto, T., Hasegawa, S., Hibi, Y., Hirao, T., Hiromoto, N., Hong, S.S., Imai, K., Ishigaki, M., Ishiguro, M., Ishihara, D., Ita, Y., Jeong, W.S., Jeong, K.S., Kaneda, H., Kataza, H., Kawada, M., Kawai, T., Kawamura, A., Kessler, M.F., Kester, D., Kii, T., Kim, D.C., Kim, W., Kobayashi, H., Koo, B.C., Kwon, S.M., Lee, H.M., Lorente, R., Makiuti, S., Matsuhara, H., Matsumoto, T., Matsuo, H., Matsuura, S., Müller, T.G., Murakami, N., Nagata, H., Nakagawa, T., Naoi, T., Narita, M., Noda, M., Oh, S.H., Ohnishi, A., Ohyama, Y., Okada, Y., Okuda, H., Oliver, S., Onaka, T., Ootsubo, T., Oyabu, S., Pak, S., Park, Y.S., Pearson, C.P., Rowan-Robinson, M., Saito, T., Sakon, I., Salama, A., Sato, S., Savage, R.S., Serjeant, S., Shibai, H., Shirahata, M., Sohn, J., Suzuki, T., Takagi, T., Takahashi, H., Tanabé, T., Takeuchi, T.T., Takita, S., Thomson, M., Uemizu, K., Ueno, M., Usui, F., Verdugo, E., Wada, T., Wang, L., Watabe, T., Watarai, H., White, G.J., Yamamura, I., Yamauchi, C., Yasuda, A.: The infrared astronomical mission AKARI*. PASJ 59, S369-S376 (2007). https://doi.org/10.1093/pasj/59.sp2.S369, 0708.1796

26. Neugebauer, G., Leighton, R.B.: Two micron sky survey - a preliminary catalog. NASA SP-3047 (1969) 
27. Neugebauer, G., Habing, H.J., van Duinen, R., Aumann, H.H., Baud, B., Beichman, C.A., Beintema, D.A., Boggess, N., Clegg, P.E., de Jong, T., Emerson, J.P., Gautier, T.N., Gillett, F.C., Harris, S., Hauser, M.G., Houck, J.R., Jennings, R.E., Low, F.J., Marsden, P.L., Miley, G., Olnon, F.M., Pottasch, S.R., Raimond, E., Rowan-Robinson, M., Soifer, B.T., Walker, R.G., Wesselius, P.R., Young, E.: The infrared astronomical satellite (IRAS) mission. The Astrophysical Journal Lettersa 278, L1-L6 (1984). https://doi.org/10.1086/184209

28. Packham, C., Telesco, C.M., Hough, J.H., Ftaclas, C.: Canaricam: the multi-mode mid-IR instrument for the GTC. In: Hidalgo-Gámez, A.M., González, J.J., Rodríguez Espinosa, J.M., Torres-Peimbert, S. (eds.) Revista Mexicana De Astronomia Y Astrofisica, vol. 24, pp. 7-12 (2005)

29. Papoular, R.: The processing of infrared sky noise by chopping, nodding and filtering. Astron. Astrophys. 117, 46-52 (1982)

30. Price, S., Walker, R., Laboratory, U.A.F.G.: The AFGL four color infrared sky survey: catalog of observations at 4.2, 11.0, 19.8, and 27.4 micrometers. AFGL-TR, Air Force Geophysics Laboratories, Air Force Systems Command, United States Air Force (1976)

31. Price, S.D., Murdock, T.L.: The revised air force geophysical laboratory infrared sky survey. AFGLTR-0208 Environemental Research papers 161, 0 (1983)

32. Rashman, M.: Terrestrial and astronomical applications of uncooled infrared technology. PhD thesis, Liverpool John Moores University. https://doi.org/10.24377/LJMU.t.00013093 (2020)

33. Rashman, M.F., Steele, I.A., Burke, C., Longmore, S.N., Wich, S., Beletic, J.: Adapting thermalinfrared technology and astronomical techniques for use in conservation biology. In: Holland, A.D. (ed.) Proc. SPIE, High Energy, Optical, and Infrared Detectors for Astronomy VIII, vol. 10709, pp. 667-676 (2018)

34. Rashman, M.F., Steele, I.A., Bates, S.D., Copley, D., Longmore, S.N.: Uncooled microbolometer arrays for ground-based astronomy. Mon. Not. R. Astron. Soc. 492(1), $480-487$ (2019). https://doi.org/10.1093/mnras/stz3497

35. Richter, M.J., Dewitt, C.N., McKelvey, M., Montiel, E., McMurray, R., Case, M.E.: EXES: the echelon-cross-echelle spectrograph For SOFIA. Journal of Astronomical Instrumentation 7(4), 1840013 (2018). https://doi.org/10.1142/S2251171718400135

36. Shaw, J., Nugent, P., Vollmer, M.: Infrared moon imaging for remote sensing of atmospheric smoke layers. Appl. Opt. 54 (2015)

37. Steele, I.A., Smith, R.J., Rees, P.C., Baker, I.P., Bates, S.D., Bode, M.F., Bowman, M.K., Carter, D., Etherton, J., Ford, M.J., Fraser, S.N., Gomboc, A., Lett, R.D.J., Mansfield, A.G., Marchant, J.M., Medrano-Cerda, G.A., Mottram, C.J., Raback, D., Scott, A.B., Tomlinson, M.D., Zamanov, R.: The liverpool telescope: performance and first results. In: Oschmann, J.M. Jr. (ed.) Proc. SPIE, GroundBased Telescopes, vol. 5489, pp. 679-692 (2004)

38. Telesco, C.M., Pina, R.K., Hanna, K.T., Julian, J.A., Hon, D.B., Kisko, T.M.: Gatircam: gemini midinfrared imager. In: Fowler, A.M. (ed.) Proc. SPIE, Infrared Astronomical Instrumentation, vol. 3354, pp. 534-544 (1998)

39. Tsang, C.C.C., Durda1, D.D., Ennico, K.A., Less, J., Propp, T., Olkin, C.B., Stern, S.A.: Small aperture airborne telescopes for planetary science. 46th Lunar and Planetary Science Conference (2015)

40. Vollmer, M., Möllmann, K.P.: Surface temperatures of the moon: measurements with commercial infrared cameras. Eur. J. Phys. 33, 1703 (2012)

41. Wenger, M., Ochsenbein, F., Egret, D., Dubois, P., Bonnarel, F., Borde, S., Genova, F., Jasniewicz, G., Laloë, S., Lesteven, S., Monier, R.: The SIMBAD astronomical database. The CDS reference database for astronomical objects. Astronomy and Astrophysics Supplement 143, 9-22 (2000). https://doi.org/10.1051/aas:2000332, astro-ph/0002110

42. Wright, E.L., Eisenhardt, P.R.M., Mainzer, A.K., Ressler, M.E., Cutri, R.M., Jarrett, T., Kirkpatrick, J.D., Padgett, D., McMillan, R.S., Skrutskie, M., Stanford, S.A., Cohen, M., Walker, R.G., Mather, J.C., Leisawitz, D., Gautier, I., Thomas, N., McLean, I., Benford, D., Lonsdale, C.J., Blain, A., Mendez, B., Irace, W.R., Duval, V., Liu, F., Royer, D., Heinrichsen, I., Howard, J., Shannon, M., Kendall, M., Walsh, A.L., Larsen, M., Cardon, J.G., Schick, S., Schwalm, M., Abid, M., Fabinsky, B., Naes, L., Tsai, C.W.: The wide-field Infrared Survey Explorer (WISE): mission description and initial on-orbit performance. Astronomical Journal 140(6), 1868-1881 (2010)

Publisher's note Springer Nature remains neutral with regard to jurisdictional claims in published maps and institutional affiliations. 